\title{
Le VIII Séminaire européen d'ethnomusicologie, Genève, septembre 1991
}

\section{Keith Howard}

Traducteur : Isabelle Schulte-Tenckhoff

\section{OpenEdition \\ Journals}

Édition électronique

URL : http://journals.openedition.org/ethnomusicologie/2477

ISSN : 2235-7688

Éditeur

ADEM - Ateliers d'ethnomusicologie

Édition imprimée

Date de publication : 1 janvier 1992

Pagination : 325-336

ISBN : 978-2-8257-0456-1

ISSN : 1662-372X

Référence électronique

Keith Howard, "Le VIII Séminaire européen d'ethnomusicologie, Genève, septembre 1991 », Cahiers d'ethnomusicologie [En ligne], 5 | 1992, mis en ligne le 15 décembre 2011, consulté le 20 avril 2019. URL : http://journals.openedition.org/ethnomusicologie/2477 


\section{RENCONTRES}

\section{Le VIII ${ }^{e}$ Séminaire européen d'ethnomusicologie, Genève, septembre 1991*}

Le Séminaire européen d'ethnomusicologie (SEEM) traverse un moment crucial de son histoire. Créé en 1981 à Belfast pour et par des ethnomusicologues professionnels, il fut d'abord présidé par feu John Blacking. Une première rencontre des membres fut organisée en 1983 à Cologne; depuis 1985, des réunions annuelles se sont tenues respectivement à Belfast, Londres, Paris, Tuczno/Pologne, Sienne et Berlin.

Organisée en septembre 1991 par les Ateliers d'ethnomusicologie au Centre de conférence de Bossey près de Genève, la huitième rencontre annuelle du SEEM réunit une centaine de participants venant des pays suivants: Allemagne, Autriche, Belgique, Biélorussie, Canada, Danemark, Espagne, États-Unis, France, Géorgie, Grande-Bretagne, Israël, Italie, Kazakhstan, Lettonie, Ouzbekistan, Pays-Bas, Pologne, Roumanie, Russie, Suisse et Ukraine. Le séminaire traita de cinq thèmes: les approches ethnomusicologiques de la polyphonie, l'esthétique, les méthodes de terrain, la Chine et l'Extrême-Orient, et le rôle de l'ethnomusicologie dans la société moderne. Pour la première fois depuis Belfast en 1985, afin de rappeler aux participants que l'ethnomusicologie ne peut jamais se borner à une recherche purement historique et scientifique, Genève avait prévu des manifestations «non scientifiques», en montrant un film de Blacking et en organisant des concerts avec des interprètes originaires du Burkina Faso, des pays andins, de Suisse, de Chine, du Japon et de Corée.

Depuis 1981, le règlement du SEEM relatif à l'admission de nouveaux membres s'est assoupli, les étudiants et ceux qui se meuvent à la périphérie de la discipline étant maintenant admissibles. Bien qu'il fût à ses débuts une association informelle, le SEEM a atteint une dimension qui exige la création d'un comité, quelle que soit sa structure. La nécessité d'une transformation hiérarchique sem-

\footnotetext{
Traduit de l'anglais par Isabelle Schulte-Tenckhoff.
} 
ble admise par la plupart des membres, mais la mort de Blacking en janvier 1990 priva le Séminaire d'un leader charismatique. Or l'ethnomusicologie s'est développé diversement selon les pays. C'est seulement par une transformation structurelle permettant d'accommoder chaque perspective ethnomusicologique que le SEEM pourra faire rayonner la discipline en Europe. Essentiellement, le SEEM est appelé à fonctionner comme l'équivalent européen de la Society for Ethnomusicology, d'obédience américaine. Voilà qui figurait déjà dans la conception originelle de Blacking. En effet, il insistait, en tant que président de la Society for Ethnomusicology au début des années 1980, sur le fait qu'elle devait principalement servir les intérêts des chercheurs américains (Blacking, in Howard 1990: 200).

Pour consolider sa position, le SEEM doit lancer une publication régulière ${ }^{1}$. Et, forte d'une transformation dont il reste à déterminer les modalités, le SEEM devrait être à même de surmonter les périodiques régionaux par ses publications, en coordonnant les associations ethnomusicologiques nationales.

Mises à part les publications, comment le SEEM peut-il accroître son efficacité? Je pense que, tout d'abord, les chercheurs européens devront s'engager autant, sinon davantage en faveur du SEEM que de la Society for Ethnomusicology ou de l'International Council for Traditional Music (ICTM). Ensuite, il nous faut quitter notre tour d'ivoire (une grande partie de la recherche ethnomusicologique reste confinée dans l'univers apparemment clos des universités européennes), afin de nous joindre à ceux qui, à nos portes, pratiquent une musique vivante ou savent l'apprécier. Ainsi pourrions-nous coopérer plus étroitement avec les médias et contribuer à façonner la perception que le public peut avoir des musiques venant du monde entier; ce qui signifie du même coup un plus grand rayonnement de notre discipline.

Le projet de transformation du SEEM va donc bien au delà de considérations relatives à l'admission de nouveaux membres. Il faut se rendre compte que dans la conjoncture musicale actuelle, les ethnomusicologues sont appelés à jouer un rôle politique. Les règles gouvernant les carrières universitaires ont changé. L'ancien point de vue, qui veut que les anthropologues (et donc les disciples de Blacking) demeurent des observateurs impartiaux, ne peut guère être défendu à l'heure actuelle: évoquons à ce sujet le dilemme ressenti face à une culture moribonde, dont Colin Turnbull parle dans Un peuple de fauves (1973); ou l'attitude colonialiste de nombreux chercheurs de terrain qui, il n'y a pas si longtemps, jetèrent les bases de notre discipline; ou encore les exemples d'abus de données anthropologiques cités dans le livre de J.A. Barne, Who Should Know What? (1979). Nous reconnaissons nos obligations morales envers les informateurs et les musiciens (même si nous n'agissons pas toujours comme si ces obligations devaient nous engager); nous commençons à mesurer notre responsabilité lorsqu'il s'agit d'éviter, dans la mesure du possible, de causer un préjudice per-

L'assemblée générale de 1991 adopta à une nette majorité une motion donnant au conseil le mandat d'élaborer un projet de publication. 
sonnel à nos audiences et à nos étudiants ${ }^{2}$. Ce que nous avons choisi d'étudier, ce que nous défendons dans notre enseignement et ce que nous publions - que ce soit sous forme sonore ou livresque - reflète nos jugements de valeur (jugements sur la qualité musicale, mais peut-être aussi sur le contexte de la musique).

Aujourd'hui plus que jamais, la musique passe par les mass médias, vis-à-vis desquels les ethnomusicologues montrent une certaine réticence. En effet, nombreux sont ceux qui critiquent les exigences de producteurs mus par des critères journalistiques ou mercantiles. D'autres ont découvert que la tentative de réaliser une émission qui soit satisfaisante pour les informateurs risque de ne pas servir les intérêts de notre propre société, ou n'atteint pas un large public. Beaucoup se méfient des projets commerciaux. D'autres pensent qu'ils pourront exercer une influence sur les décisions d'impresarios, ou arguent que les entreprises commerciales permettent d'en soutenir d'autres, sans buts commerciaux, qui portent à la connaissance publique des traditions musicales plus «authentiques».

En 1981, l'année où le SEEM organisa sa première rencontre, l'International Folk Music Council (IFMC) devint l'International Council for Traditional Music (ICTM). Désormais, une certaine idée de la nature même de l'ethnomusicologie semblait assurée, du moins pour quelque temps. Or le concept de tradition - et, par voie de conséquence, celui d'authenticité - est trompeur. Avant 1981, des définitions possibles de «musique populaire» étaient l'objet de tortueux débats ( $c f$. Elbourne 1976), visant le plus souvent à remettre en cause la définition provisoire adoptée en 1954 par l'ICTM:

«La musique populaire est le produit d'une tradition musicale qui s'est développée grâce à la transmission orale. Les facteurs contribuant à forger la tradition sont: I) la continuité reliant le présent au passé; II) la variation, produite de l'impulsion créatrice de l'individu ou du groupe; et III) la sélection par la communauté, déterminant la ou les formes sous lesquelles la musique survit... Le terme ne recouvre pas la musique populaire composée, qu'une communauté reprend en tant que telle et sans y apporter de changement, car c'est le refaçonnage et la re-création de la musique par la communauté qui lui confère son caractère populaire» (Anon. 1955: 23).

Lors du débat, peu de cas spécifiques furent cités. Certains ethnomusicologues acceptent les défauts d'un disque comme celui de David Fanshawe, African Sanctus (Philips, 6558 001, 1975): là, les musiciens locaux semblent ne pas avoir eu leur mot à dire sur le produit final, ni avoir obtenu leur part des redevances. [Citons toutefois un extrait de la critique de sa réédition en CD (1991) par Laurent Aubert, paru dans Le monde de la musique (151, 1992, p. 118), qui remet les choses en place: «...ce bric-à-brac hétéroclite atteste les pérégrinations d'un habile chasseur de sons [...] Présenté autrement, il aurait fourni une documentation précieuse sur certaines traditions musicales d'Afrique orientale [...] Mais l'intention sous-jacente dérange: ne serait-il pas plus estimable, lorsqu'on a eu accès à de telles merveilles, de s'effacer devant ses découvertes?» (n.d.l.r.)]. Nombreux sont aussi ceux qui se plaignent du contrôle que des festivals européens comme WOMAD et certains labels exercent sur la vente de la musique du tiers monde aux consommateurs européens, tout en en tirant des profits substantiels. 
Les auteurs de ce texte, Cecil Sharpe et d'autres, étaient partis d'une conception inadéquate du processus du changement culturel; étant donné l'importance toujours plus grande que la «world music» revêt en Europe, une telle définition paraît fort naïve. Si la justification de notre recherche réside avant tout dans l'analyse de l'homme en tant que faiseur de musique, il nous faut persister à explorer les modes d'utilisation de la musique. Nous ne pouvons pas nous permettre de laisser notre propre goût musical altérer notre perspective. Au milieu des années cinquante, les mass médias étaient à peine présents; aujourd'hui ils sont l'agent principal de la transmission musicale à l'échelle mondiale. Pour assurer la pérennité de l'ethnomusicologie en tant que discipline, nous devons non seulement recourir à la tradition orale, morte ou en voie de disparition, mais encore élaborer des stratégies pour aborder les musiques que l'on entend aujourd'hui.

De nombreux genres appelés «fusion» ou, euphémiquement, «world music» ou "world beat », ont des origines qui ne sont pas étrangères à la notion de «tradition». En limitant notre angle d'approche à ce que nous percevons comme des formes «authentiques» ou «traditionnelles», nous opérons une sélection qui rappelle celle pratiquée par les musicologues travaillant sur la musique classique occidentale. Cela n'est pas digne de l'ethnomusicologie, qui seule s'occupe de tout l'univers de la musique:

«[Nous aimerions] nouer des liens plus étroits entre les découvertes de l'ethnomusicologie et les préoccupations de la musicologie en général. Le compartimentage de la musicologie me semble être une tragédie. Je pense que Guido Adler n'a jamais souhaité un tel développement lorsqu'il s'est joint à Chrysander et Spitta pour fonder la Vierteljahrschrift für Musikwissenschaft (1884) [...] Je crois que nous serons bientôt en mesure de supprimer le préfixe d'«ethno»dans ethnomusicologie. Nous aurons alors reconquis une musicologie unifiée, véritablement fertilisée par l'apport de l'ethnomusicologie” (Blacking, in Howard 1990: 199-200).

A Genève, une table ronde réunie autour du thème «Les applications de l'ethnomusicologie dans la société moderne" consacra une journée à ces questions. Le programme définissait le thème de la manière suivante:

«L'extension des musiques dites traditionnelles au delà de leur contexte originel a enrichi la culture musicale de beaucoup; mais elle risque également d'engendrer des représentations erronées d'autres cultures et des pratiques musicales déformées».

L'idée du caractère déformateur des médias était largement répandue, et des jugements pseudo-scientifiques sur les «musiques dites traditionnelles» décourageaient tout examen du marché musical actuel.

Tout d'abord Robert Günther (Cologne), dans un exposé intitulé «L'ethnomusicologue dans la société contemporaine», se mouvait sinueusement entre des définitions démodées et la praxis musicale actuelle. Il commença en affirmant que l'ethnomusicologie ne détient ni stratégie ni objectif préétabli et que les ethnomusicologues établissent un lien entre producteur (=musicien) et 
récepteur (=auditeur). L'ethnomusicologue est un médiateur appelé à mettre en œuvre son savoir-faire, sa compétence et sa personnalité d' «agent responsable». $\mathrm{Au}$ début, Günther échappa à la politisation, en affirmant que la volonté du chercheur, «le fait qu'il manifeste un intérêt envers toute musique qu'il entend» était la clef pour atteindre ses objectifs. Derrière tout cela se profilait toutefois ce que Günther appelait un "point de vue personnel sur les conséquences de l'application ethnomusicologique ", exigeant de porter un jugement sur l'authenticité et la tradition. Plus tard, Chérif Khaznadar, directeur de la Maison des cultures du mondes à Paris et membre du Comité pour les arts extra-européens (CAEE) explicita ce qu'il fallait entendre par un tel jugement, lorsqu'il évoqua les dangers que récèle la tendance actuelle à transformer la musique pour faciliter sa commerciaiisation. Khaznadar voit dans la «world music» une nouvelle forme de colonialisme; étant donné que «les impresarios commerciaux sont surtout attirés par le sensationnel et l'exotique, et que le succès commercial est leur souci principal», la «world music» permet de vendre les vedettes de l'Orient «comme des petits pains».

La question reste ouverte. Frans de Ruiter, directeur du Conservatoire de La Haye et, lui aussi, porte-parole du CAEE, avança qu'aucune ligne de démarcation n'avait encore pu être tracée entre musiques traditionnelles et genres contemporains. Il se demanda comment agir face aux formes adaptées: faut-il considérer un genre millénaire comme plus «authentique» que son équivalent vieux d'un siècle seulement? En réponse, Frank Kouwenhoven (Leiden) ne voyait pas de problème à encourager à la fois la «world music» populaire et les genres régionaux «authentiques», moins accessibles; il donna comme exemple la pratique des éditeurs de textes universitaires peu rentables, qui consiste à réaliser des bénéfices en publiant des livres de cuisine à succès. De Ruiter rétorqua qu'il n'y a pas de profit à tirer de l'art, et Peter Crowe (Bordeaux) renchérit sur les propos de Khaznadar: «D'abord nous avons pris leurs minerais, puis leur nourriture, maintenant nous leur volons leur âme».

De toute évidence, c'est une question sujette à controverse, d'autant que les ethnomusicologues ne manquent sûrement pas de soutenir les cultures musicales et les musiciens avec lesquels ils travaillent. D'un côté, comme le dit Wim van Zanten (Leiden), les chercheurs peuvent aider les impresarios en Europe en leur recommendant des musiques et des musiciens locaux. Le Séminaire apprit que le CAEE voudrait même aller plus loin, en recourant à des musicologues indigènes afin d'assurer de bons choix (mais je doute qu'il existe beaucoup de musicologues indigènes sans idées préconçues). D'un autre côté, affirma de Ruiter, le CAEE savait que l'exportation de genres locaux exigeait une adaptation à l'oreille européenne. Ainsi discuta-t-il brièvement de solutions possibles à des problèmes comme la durée du concert, l'aménagement de la scène, voire l'intelligibilité ou l'accessibilité de musiques particulières (différences dans le système tonal, harmonisation, contenu religieux).

Citant le cas de musiciens mongols en tournée en Grande-Bretagne, Carole Pegg (Cambridge) proposa qu'à l'instar des médiateurs de Günther, les ethnomusicologues assument en partie la responsabilité de faciliter un échange. Il revien- 
drait donc à l'ethnomusicologue de contrecarrer l'héritage colonialiste, en évitant de chercher à profiter des musiciens avec lesquels il travaille: «il faut que l'accueil d'interprètes transplantés dans un environnement qui leur est étranger, soit chaleureux et organisé avec soin ${ }^{3}$. Cela parut tout à fait raisonnable aux participants, mais à y regarder de plus près, la chose est plutôt curieuse, car la nature de l'exercice ethnomusicologique consiste précisément à profiter de la musique d'autrui: les ethnomusicologues ont un emploi grâce à leur savoir et à leur expertise.

Sylvia Delorenzi-Schenkel (Lugano) défendit son rôle de médiatrice dans une communication intitulée «L'extension de la musique dite traditionnelle par les médias d'un côté, et par les enfants d'émigrants de l'autre». Elle offrit deux récits. Dans l'un elle décrivit comment les enfants suisses de diverses origines ethniques échangeaient des chansons. L'auteur suggéra, pour faciliter la compréhension interculturelle, d'enrichir tout cela par la transmission formelle d'informations d'ordre socioculturel. Le second récit était plus intéressant, car il tenait compte de diverses approches de la musique elle-même, soit de type ethnomusicologique, soit dans le cadre des mass médias. Depuis le milieu des années quatre-vingts, Delorenzi-Schenkel produit des émissions de «musique dite ethnique» pour la radio suisse-allemande. Ses propres objectifs - réaliser un programme spécial réunissant diverses musiques traditionnelles, susciter l'intérêt des auditeurs en les mettant au défi d' «ouvrir esprit et oreilles»-divergeaient de ceux des producteurs désireux d'introduire un nouveau style de programme comprenant de la musique exotique pour divertir un large public. Initialement, on était naïf de part et d'autre: les programmateurs n'avaient qu'une vague idée de la musique non occidentale, ne pouvant s'appuyer que sur un petit nombre d'enregistrements commerciaux; pour sa part, Sylvia était convaincue des avantages d'une multiplication d'univers sonores locaux et régionaux. L'utilisation qu'elle faisait d'enregistrements réalisés sur le terrain et de musiques aborigènes ne tarda pas à provoquer des controverses, les grands pontess des médias se plaignant que pareils enregistrements passaient à côté du large public.

Tout en admettant que son audience était peu nombreuse, DelorenziSchenkel défendit son choix en rappelant le courrier d'auditeurs enthousiastes. De nombreux participants à la séance sympathisaient avec elle, car des problèmes semblables se posent dans d'autres aventures tentées par des ethnomusicologues dans les mass médias. Mais je me demande pour combien de temps les ethnomusicologues incapables d'atteindre un large public pourront maintenir intacte leur intégrité. Je viens de réaliser pour la $\mathrm{BBC}$ deux émissions de radio sur la musique coréenne. L'une et l'autre seront probablement diffusées une fois, à un moment de faible écoute, puis elles tomberont dans l'oubli. Il est difficile de justifier le prix et l'effort que coûte chaque programme lorsqu'on le mesure, par exemple, à la popularité de l'émission hebdomadaire de Kershaw sur la «world music».

3 Cité d'après le résumé de la séance no. 21 de la réunion. 
Des collègues venus des anciens États est-européens énoncèrent un point de vue différent lors de la table ronde. Chacun d'eux adhérait au principe que l'ethnomusicologue est appelé à s'impliquer directement dans les décisions relatives à la qualité, à l'authenticité et à la nature des enregistrements ou des concerts; implicitement, chacun se sentait maintenant en mesure de réagir contre le totalitarisme (culturel) russe. Slawomira Kominek (Varsovie), avec l'exemple de la minorité lithuanienne en Pologne, affirma qu'au sein de la musique populaire, le chant et la danse sont utiles pour développer une nouvelle identité ethnique ou nationale. Mais elle releva que le «folklorisme musical» qui en résulte est stylisé et tend à accentuer l'exotique, car il simplifie les sons musicaux et les images symboliques. A partir de là, Zinaida Mozheiko (Minsk) émit un avertissement; de son point de vue, les tentatives visant à créer un environnement de «tradition authentique» risquaient d'entraîner une standardisation susceptible de porter atteinte aux images ethniques. Elle affirma que pareilles tentatives étaient souvent encouragées par des sponsors commerciaux désireux de rendre les prestations attractives plutôt qu'authentiques, et par des minorités isolées, car ces tentatives facilitaient la conservation de ce qui était ancien, tout en décourageant le changement économique.

Boris Avramets (Riga), en revanche, soutint que les matériaux folkloriques nationaux permettaient l'intégration des ethnies en un réseau global de liens culturels. Les ethnomusicologues pourraient contribuer à l'indépendance lettone en faisant revivre la musique ethnique et en en restaurant les contextes socioculturels. Anna Czekanowska (Varsovie) poursuivit dans la même veine, en avançant, à partir de son étude de l'éducation musicale étatique au Kazakhstan, que l'identification avec une culture musicale spécifique aide à découvrir l'ethnicité. Les mêmes problèmes se retrouvent à travers le monde entier: peut-être le moment et l'opportunité sont venus d'amener les ethnomusicologues de l'ouest et ceux de l'est à échanger leurs expériences et à élaborer des stratégies communes. Les intervenants ont bien fait ressortir l'urgence du problème, et nous avons effectivement un rôle à jouer dans la redécouverte des identités culturelles régionales là où la Russie a longtemps gouverné.

Un échange plus intense entre Européens occidentaux et orientaux au sein du SEEM donne lieu à de nouvelles approches et à de nouvelles méthodes. A Genève, dix-sept interventions venaient de collègues est-européens. Au delà des questions posées par la table ronde, on constata deux approches typiques qui s'opposaient - quoique jamais exclusivement - à la pratique habituelle des chercheurs ouest-européens.

Dans un cas, les musicologues indigènes associaient recherche et éducation, en vue d'étudier leur propre musique. Dans l'autre, des ethnomusicologues se vouaient, en tant que «scientifiques», à l'élaboration de théories globales, voire interculturelles sur la musique et l'identité. La première approche était suivie par Edisher Garakanidse (Tbilisi) dans sa communication sur les dialectes musicaux de Géorgie; par Ewa Dahlig (Varsovie) dont la communication sur la vièle polonaise, décrivant avec force détail comment les bourdons rythmiques homophones passent à l'hétérophonie, lança un défi aux théories des musicologues 
polonais ${ }^{4}$; par Saule Utegaliyeva (Alma-Ata) qui traita de la polyphonie dans la musique des peuples turcs; enfin par Yelena Pushkaryova (Moscou) dans son étude des chants personnels des Nenet.

La seconde approche, d'ordre plus globale, adoptée par Izaly Zemtsovsky (Saint-Pétersbourg), Iosif Zhordania (Tbilisi), Helen Vassyltchenko (Moscou), Margarita Karatygina (Moscou) et d'autres, court néanmoins le risque de favoriser des images déformées de cultures particulières. En partant du terme grec d'agon, Zemtsovsky développa la notion d'antiphonie, considérée comme une forme de dialogue entonné. Affirmant que le langage plonge ses racines dans l'échange de sons, il montra les relations entre le chant et les termes désignant le conflit ou la bataille parmi les Turcs, Yakout, Roumains, Inuit, Slaves, Finnois, ainsi que dans les cultures méditerranéennes et moyen-orientales. La démonstration de Zemtsovsky ne faisait nullement référence à la notion, désormais rejetée, de diffusion; elle visait plutôt à décrire trois transitions possibles du chant monodique à la polyphonie, soit: les signaux, l'appel suivi d'une réponse, et la polyphonie intégrée. Or l'argument demeurait faible, d'abord parce que Zemtsovsky dit bien que les concepts de monodie et de polyphonie traduisaient une dichotomie hypothétique sans correspondance dans la réalité, et deuxièmement parce qu'il ne tint pas compte de la dimension émique de la question.

Zhordania proposa une vue plus évolutionniste du même thème. Il affirma que la plupart des groupes caucasiens pratiquaient des formes polyphoniques similaires, tout en se différenciant sur le plan religieux et linguistique; il en conclut que la polyphonie représente une forme d'expression fort ancienne ayant survécu aux processus migratoires - affirmation qui contredit les théories diffusionnistes: Curt Sachs aurait sûrement manifesté son désaccord. Zhordania justifia son interprétation par un recours à la génétique: conçevant, comme Zemtsovsky, le dialogue comme la base de la polyphonie, il le mit en rapport avec le développement du langage. Il déclara notamment que les Asiatiques ont acquis le langage articulé il y a 400000 ans, ce qui expliquerait pourquoi les découvertes archéologiques font état d'un élargissement subit du volume du cerveau; les Caucasiens aurait suivi, il y a 100000 ans à peine. Zhordania en déduisit que la polyphonie remonterait aux trois mille siècles supplémentaires vécus sans langage. Pour ma part, je rétorquerais que la primogéniture asiatique peut être datée, grâce notamment à la manipulation japonaise des données. Lors de la rencontre du SEEM en 1990, Max Peter Baumann tomba dans un piège semblable lorsqu'il démontra de manière convaincante (mais erronée?)

4 Cette phrase explique pourquoi j'ai placé «scientifiques» entre guillemets. Je reconnais la volonté de certains d'affirmer le caractère scientifique de l'ethnomusicologie, mais j'estime que le jeu musical est un art. Nombreux sont encore ceux qui, comme Dahlig, souscrivent jusqu'à un certain point au principe de la bimusicalité en ethnomusicologie, qui remonte à Mantle Hood: pour eux, le jeu musical représente une part vitale de leur travail. John Blacking, qui était pianiste, aurait défendu l'art: il enseigna l'anthropologie et l'ethnomusicologie dans le cadre de la Faculté des Lettres à Belfast (Howard 1990: 195, 197). 
que les Japonais étaient plus musicaux, car la sphère droite de leur cerveau était mieux développée (1990: 52-76, d'après Tsunoda 1985).

Karatygina aborda la problématique du genre musical, avec l'exemple des cultures nomades. A partir de l'hypothèse que le genre fonctionne comme le modèle des systèmes humains, elle présenta une «nouvelle» approche multi-disciplinaire dans laquelle il faut voir le genre comme un phénomène culturel entretenant des liens spécifiques avec l'organisation sociale, comme elle tenta de le démontrer en comparant diverses cultures musicales. Veit Erlmann (Berlin) critiqua cette théorie dans laquelle il ne voyait rien de particulièrement nouveau: l'analogie entre le genre et son contexte culturel aurait fait l'objet de nombreuses études en Amérique au cours des vingt dernières années; l'ouvrage d'Anthony Seeger, Why Suya Sing (1987), en étant un exemple parlant. Carole Pegg s'attaqua à la comparaison: Karatygina avait utilisé un exemple mongol qui n'est interprété que par un petit groupe occidental, et non pas par les Khalka dominant, comme elle l'affirmait; tout cela démontrait clairement combien il est nécessaire de tenir compte de l'ethnicité.

La communication de Vassyltchenko, visant à démontrer le rapport entre la voix et les instruments en tant que modes respectivement primaire et secondaire de la pensée musicale, était également compromise par des erreurs de détails. Vassyltchenko affirma que, sur le pourtour de la Méditerrannée et en Inde du Sud, les instruments étaient subordonnés à la voix. Ses exemples asiatiques avaient pour fonction d'illustrer la nature symbolique des instruments: on dit ainsi que les tambours chamaniques abritent des esprits animaux, que les pierres en Asie du sud-est renferment les mânes des ancêtres. Voilà des généralisations susceptibles d'être contredites par des exemples concrets. Mais Vassyltchenko ne tint pas non plus compte des perceptions étiques. Ainsi la place centrale de l'empereur dans la pensée chinoise était-elle laissée de côté en faveur de considérations saisonnières. En Corée, les termes d'origine chinoise relatifs à la musique de cour étaient présentés comme provenant du folklore indigène. A Java, le le terme de gung (pour gong) était dérivé selon un procédé fort complexe de gunung, montagne, sans considérer qu'il pourrait s'agir d'une onomatopée. Et Confucius, dont on dit qu'il passa sept ans à jouer un seul ton sur la cithare, était implicitement exclu de la démonstration par le commentaire suivant: «les instrumentistes ne deviennent jamais des héros culturels».

Je ne veux pas écarter d'emblée de telles théories générales. On peut attribuer leurs défauts en partie à l'univers théorique de la Russie ${ }^{5}$, mais aussi au fait que les chercheurs russes avaient peu accès au terrain et à la littérature adéquate. Vu la disparition réelle des cultures minoritaires, il n'en convient pas moins de repenser, en Europe occidentale, le penchant pour l'étude détaillée d'une culture locale à l'encontre, précisément, de la perspective évolutionniste - penchant manifeste chez ceux qui se sont formés à l'anthropologique britanni-

Un volume intitulé Problems of Terminology in Asian, African and American Musical Cultures fut publié à Moscou en 1990. Les communications présentées à Genève par Karatygina et Vassyltchenko puisent une bonne partie de leurs données dans les dix essais de ce volume. 
que ou à la sociologie française. Les chercheurs ouest-européens ne sont pas encore parvenus à développer des théories musicales qui soient valables à l'échelle transculturelle. Or, quelle que soit la valeur intrinsèque de telles théories, il ne faut pas négliger les interrogations générales au profit de descriptions détaillées. Mais pour permettre à l'ethnomusicologue de se profiler comme animal politique, que ce soit en tant que médiateur, promoteur ou producteur dans les mass médias, il ne faut pas non plus que ces descriptions servent de base au traitement de questions générales ${ }^{6}$. Le défi le plus important que le SEEM doit actuellement relever consiste peut-être à jeter un pont entre la recherche de l'ouest et celle de l'est.

Deux communications peuvent ici servir d'exemple. L'une est due à Susanne Ziegler (Berlin) qui parla de son étude des enregistrements analytiques de polyphonies géorgiennes, réalisés dans les années trente sur trois phonographes parallèles par Gippius, Ewald et Emsheimer. En utilisant des exemples tirés de sa propre enquête de terrain en 1991 dans la région d'origine des chants, elle opposa l'ancien style (richesse des harmonies, indépendance des parties, éléments d'improvisation) au nouveau (mélodies et harmonies fixes, tonalité, manque d'individualité). Il aurait été intéressant de découvrir comment ses collègues géorgiens (Zhordania et Garakanidse) expliquent de telles différences mais, hélas, l'échange international au sein du SEEM était trop fragile pour favoriser pareille discussion.

La seconde communication à mentionner dans ce contexte est celle de Sylvie Bolle-Zemp (Paris), qui traita également des chœurs géorgiens, en comparant notamment les groupes traditionnels d'amateurs et les ensembles folkloriques contemporains. Elle fit mention de l'«illusion d'un vaste pool d'authenticité» et relata comment les amateurs locaux se trouvaient graduellement remplacés par des choristes qui imposaient aux chants une ornementation plus régulière, une intonation plus stable, des tempos plus rapides et, selon les directeurs des chœurs, une plus grande clarté. Dans une tentative de démontrer les mécanismes de transformation des représentations de la vie rurale, Bolle-Zemp offrit une comparaison entre la Suisse et la Géorgie. Bernard Lortat-Jacob (Paris) et Veit Erlmann lui rétorquèrent que la folklorisation implique une transformation en produit de consommation, si bien que chaque nation détient moins un héritage primitif qu'une ou deux réponses affirmant la capacité de résistance des gens au productivisme et, de ce fait, leur autonomie.

Les six communications présentées à la séance consacrée à la Chine et à l'Extrême-Orient illustrèrent une fois de plus la nécessité, dans le cadre de

6 Un compte rendu récent de A Commonsense View of All Music critique Blacking pour s'être trop inspiré du cas des Venda: «il est inadmissible que dans un iivre voué à un examen des fondements et des universaux musicaux, le matériau illustratif soit vendacentrique» (Josephson 1991: 268). Je pense que la même remarque vaut pour les extraits de Dancing de Blacking, visionnés à Genève; il s'agit d'une série de films réalisés pour la télévision d’Ulster, où Blacking semblait plus proche de ses matériaux africains que de la discothèque occidentale. 
l'étude d'une région particulière, de ne pas perdre de vue les interrogations générales de l'ethnomusicologie ${ }^{7}$. La séance en question fut organisée par la Fondation européenne pour la recherche sur la musique chinoise (Chime). Cinq communications portaient sur la Chine: celles de Dai Xiaolian (Leiden), Frank Kouwenhoven (Leiden), François Picard (Paris), Jonathan Stock (Belfast) et Huang Bai (Leiden). La sixième, d'Ingrid Fritsch (Bonn), rassemblait de nombreuses informations actuelles et historiques sur les goze, musiciennes japonaises aveugles maintenant en voie de disparition. Bien conçu, l'exposé de Stock sur les techniques de construction du jeu de la vièle à deux cordes parut convaincant - ce qu'il n'était pas en réalité, car Stock contourna soigneusement deux préoccupations récentes des ethnomusicologues de Belfast: d'une part, l'étude menée par Blacking et Baily sur les mécanismes biologiques déterminant les techniques de modélisation et la construction mélodique du jeu instrumental; de l'autre, la représentation informatisée des types de modélisation selon Kippen. Quant à Kouwenhoven, qui présenta une composition unique de He Luding datant de 1934, Cowherd's Flute, il chercha à défendre son idée que «la plupart des compositeurs chinois n'ont pas réussi à trouver de solution satisfaisante dans leurs tentatives pour adopter la tonalité occidentale». Or Kouwenhoven tenait pour vraie sa propre perception de la tonalité occidentale (illustrée malheureusement à l'aide d'une variante à cordes particulièrement romantique de la pièce en question), sans tenir compte de la manière dont les Chinois perçoivent le recours à des éléments musicaux occidentaux. Il négligea ainsi, à l'instar de nombreux intervenants est-européens, le point de vue émique. Ce problème, notamment dans le cas de la musique indonésienne, a récemment fait l'objet d'un débat impliquant Jody Diamond et d'autres dans EthnoFORUM. Pour ce qui est de la musique chinoise, il élargit le cadre des discussions qui eurent lieu en 1979, lors du Festival de musique orientale de Durham ${ }^{8}$.

Pour conclure, si l'on peut, à juste titre, témoigner d'un rayonnement plus large de l'ethnomusicologie, celle-ci est encore loin de s'être établie comme discipline. En termes méthodologiques, de nombreux défis sont à relever par les Européens de l'ouest comme de l'est, en vue de la création d'une base commune et d'études particulières et détaillées enrichies par une perspective transculturelle. D'autre part, l'ethnomusicologie semble toujours confinée dans une position précaire, si l'on songe à son rôle dans l'université (voir le nombre de membres du SEEM n'ayant pas participé à nos rencontres depis plusieurs années) ou à son influence limitée sur la production de disques commercialisés et de pro-

7 Il paraît qu'Amnon Shiloah (Jérusalem) et Jean During (Paris) ont su démontrer les avantages d'un angle d'approche plus serré. Malheureusement, l'organisation de séances parallèles m'empêcha d'assister à la présentation de ces communications consacrées à l'esthétique des musiques arabe et persane, ainsi qu'au débat animé qu'elles suscitèrent.

$8 \quad C f$. Robert Provine, Fang Kun et Thrasher dans Asian Music 12(2)/1981. 
grammes médiatiques. Il faut que les ethnomusicologues élaborent des stratégies pour agir efficacement comme médiateurs et promoteurs des cultures musicales. Répétons-le: l'élargissement du SEEM est bienvenu, mais le Séminaire doit œuvrer en faveur d'une transformation se concrétisant par l'établissement d'une structure organisationnelle et d'un programme précis, afin de permettre aux Européens de relever ensemble les défis qui se présentent à eux.

Keith Howard

\section{Bibliographie}

Anon.

1955 «Definition of folk music». Journal of the International Folk Music Council 7.

BARNES J.A.

1979 Who Should Know What? Harmondsworth: Penguin.

BAUMANN Max Peter

1990 «Das Ohr als Erkenntnisorgan: Prolegomenon zur Anthropologie des Hörens». In: VIIth European Seminar in Ethnomusicology: Pre-publication of the Conference Papers. Berlin: International Institute for Comparative Music Studies/Documentation/Abteilung Musikethnologie des Museums für Völkerkunde, p. 47-62.

ELBOURNE R.P.

1976 «The question of definition». Yearbook of the International Folk Music Council 7: 9-29.

HOWARD Keith

1990 «Un homme musical: entretien avec John Blacking». Cahiers de musiques traditionnelles 3: 187-201.

JOSEPHSON David

1991 «Review of John Blacking, 'A Commonsense View of All Music': Reflections on Percy Grainger's contribution to ethnomusicology and music education ». Ethnomusicology 35(2): 263-68.

SEEGER Anthony

1987 Why Suya Sing. A Musical Anthropology of an Amazonian People. Cambridge: Cambridge University Press.

TURNBULL Colin

1973 Un peuple de fauves. Paris: Stock. 